\title{
Does Quantitative Electroencephalography Refine Preoperative Cognitive Assessment in Parkinson's Disease Patients Treated with Deep Brain Stimulation? A Follow-Up Study
}

\author{
Christian Saleh Antonia Meyer Menorca Chaturvedi Selina Beltrani \\ Ute Gschwandtner Peter Fuhr
}

Department of Neurophysiology and Neurology, University Hospital Basel, Basel, Switzerland

\section{Keywords}

Quantitative electroencephalography · Deep brain stimulation · Parkinson's disease - Cognitive decline · Neuropsychological testing $\cdot$ Screening

\begin{abstract}
Objective: Deep brain stimulation (DBS) in Parkinson's disease (PD) is associated with an increased risk of post-operative cognitive deterioration. Preoperative neuropsychological testing can be affected and limited by the patient's collaboration in advanced disease. The purpose of this study was to determine whether preoperative quantitative electroencephalography (qEEG) may be a useful complementary examination technique during preoperative assessment to predict cognitive changes in PD patients treated with DBS. Methods: We compared the cognitive performance of $16 \mathrm{PD}$ patients who underwent bilateral subthalamic nucleus DBS to the performance of 15 PD controls (matched for age, sex, and education) at baseline and at 24 months. Cognitive scores were calculated for all patients across 5 domains. A preoperative 256-channel resting EEG was recorded from each patient. We computed the global relative power spectra. Correlation and linear regression models were used to assess associations of preoperative EEG measures with postoperative cognitive scores. Results: Slow waves (relative delta and theta band power) were negatively correlated with
\end{abstract}

karger@karger.com www.karger.com/dem

Karger $\%$
(C) 2021 The Author(s)

Published by S. Karger AG, Basel

This is an Open Access article licensed under the Creative Commons Attribution-NonCommercial-4.0 International License (CC BY-NC) (http://www.karger.com/Services/OpenAccessLicense), applicable to the online version of the article only. Usage and distribution for commercial purposes requires written permission. post-operative cognitive performance, while faster waves (alpha 1) were strongly positively correlated with the same scores (the overall cognitive score, attention, and executive function). Linear models revealed an association of delta power with the overall cognitive score $(p=0.00409$, adjusted $\left.R^{2}=0.6341\right)$. Verbal fluency (VF) showed a significant decline after DBS surgery, which was correlated with qEEG measures. Conclusions: To analyse the side effects after DBS in PD patients, the most important parameter is verbal fluency capacity. In addition, correlation with EEG frequency bands might be useful to detect particularly vulnerable patients for cognitive impairment and be supportive in the selection process of patients considered for DBS.

(C) 2021 The Author(s)

Published by S. Karger AG, Basel

\section{Introduction}

Parkinson's disease (PD) is a progressive neurodegenerative disease characterized by motor and non-motor manifestations $[1,2]$. PD has long been considered primarily a motor disease with the cardinal symptoms of tremor at rest, rigidity, akinesia, and postural instability, upon which diagnosis and treatment are based [2-4].

However, the non-motor manifestations of PD have gained increasing recognition [3]. Non-motor manifestations generally constitute the prodromal phase of $\mathrm{PD}$ years 
Table 1. Values represent the median and interquartile range

\begin{tabular}{llll}
\hline & PD patients with DBS & PD patients without DBS & $p$ value \\
\hline$n$ & 16 & 15 & $\mathrm{~ns}$ \\
Age, years & $66(63,68.5)$ & $64(63,68.5)$ & $\mathrm{ns}$ \\
Gender (female) & 5 & 4 & $\mathrm{~ns}$ \\
Education & $14(12,16.5)$ & $14(12,17)$ & $\mathrm{ns}$ \\
MMSE & $28(28,29.5)$ & $29(28.5,30)$ & $\mathrm{ns}$ \\
Disease duration & $8.5(3.5,13.5)$ & $6(4,9)$ & $\mathrm{ns}$ \\
LED & $709.5(442.75,1,560)$ & $653(477.5,760)$ & $\mathrm{ns}$ \\
UPDRS-II & $8(8,11.25)$ & $8(5,10)$ & $\mathrm{ns}$ \\
UPDRS-III & $17(7.75,20.25)$ & $13(10,19.5)$ & $\mathrm{ns}$ \\
UPDRS-IV & $4(1.75,4.75)$ & $2(1,4)$ & $\mathrm{ns}$ \\
Duration of FU, months & $24.50(16.50,40)$ & $24(23,25.5)$ & \\
\hline
\end{tabular}

DBS, deep brain stimulation; PD, Parkinson's disease; MMSE, Mini-Mental Status Examination; UPDRS-II-IV, Unified Parkinson's Disease Rating Scale (tested in on status); LED, levodopa equivalent dose; FU, follow-up.

before the onset of the motor phase [4-6]. Cognitive impairment is a common non-motor manifestation of $\mathrm{PD}$ with a significant impact on the quality of life [7-10]. Firstline treatment for PD consists of pharmacological treatment. Potential drawbacks of medical treatment are dyskinesias or disease refractory to pharmacological management. Deep brain stimulation (DBS) is a surgical remedy for medically intractable PD. The 2 main DBS targets for PD are the subthalamic nucleus (STN) and the globus pallidus internus. Although there is no consensus on the optimal target choice $[11,12]$, there is a broad consensus that dementia is a clear contraindication for DBS, but there is no consensus with regards to mild cognitive impairment. DBS has proven to be an effective treatment for the motor symptoms of $\mathrm{PD}$, but post-operative cognitive decline is recognized as a potential complication, despite thorough preoperative neuropsychological testing [13-17]. Thus, it is of critical importance to identify additional and reliable examination techniques to predict the post-operative cognitive course of patients considered for DBS. Although electroencephalography (EEG) is considered a useful method to predict cognitive decline in PD [18-23], it is not a component of the standard preoperative evaluation for DBS [24]. A background rhythm frequency that is below the median value (i.e., $<8.5 \mathrm{~Hz}$ ) has been found to be associated with a higher incidence over time of cognitive impairment in PD [18-24]. The objective of our study was to determine whether any aspects of the preoperative quantitative EEG correlate with the post-operative cognitive state in PD patients treated with STN-DBS and therefore potentially provide a complementary screening technique to increase selection accuracy of patients considered for DBS.

\section{Patients and Methods}

\section{Demographics}

Twenty-seven patients were initially included in our study. One male patient was excluded when his DBS system had to be explanted because of a brain abscess. Twenty-six patients underwent baseline neuropsychological testing.

Twenty-six patients had a preoperative EEG. Ten patients were excluded from the final analysis ( 3 women and 7 men) for artefact contamination (sleepiness/sleep, motion artefacts). Thus, 16 patients (5 women and $11 \mathrm{men}$ ) were included in the final analysis. These were matched to $15 \mathrm{PD}$ patients treated conservatively (i.e., without DBS) for age, gender, education, Mini-Mental StatusExamination (MMSE), disease duration, levodopa equivalent dose (LED), and Unified Parkinson's Disease Rating Scale (UPDRS) II-IV (Table 1). The control group (patients from our clinic) did not differ for age, gender, education, MMSE, LED, UPDRS, disease duration, and follow-up.

\section{DBS Surgery}

All patients with $\mathrm{PD}$ who undergo evaluation for DBS by our DBS team are eligible for participation in the study. Whether or not DBS is indicated for each patient is determined by the team based on clinical criteria alone, and this determination does not form a part of the study. Patients are selected for DBS treatment based on the established criteria modified [25] after Coleman and Ostrem [26].

Inclusion Criteria for the DBS Group

1. Idiopathic PD according to the UK Brain Bank Criteria

2. Motor impairment despite state-of-the-art therapy

3. Robust improvement on L-dopa ( $>30 \%$ in the UPDRS-III score except tremor)

4. Awareness of risks and realistic expectations of surgical outcome by the patient

\section{Exclusion Criteria for the DBS}

1. Comorbidity with any other neurological or psychiatric disease

2. Mental incompetence to provide informed consent to participate in the study (MMSE <24) 
3. Contraindications for DBS seen in MRI scan

Stimulating electrodes were stereotactically implanted into the STN. Patients were awake for the deep brain procedure. In the second part of the procedure performed under general anaesthesia, the permanent electrodes were connected to a subcutaneously implanted impulse generator, which was usually placed superficial to the pectoralis fascia just inferior to the clavicle.

\section{Neuropsychological Assessment}

The neuropsychological assessment has been described in detail in a previous publication [24]. Briefly, the patients completed a comprehensive battery of neuropsychological tests. They were tested preoperatively on medications and post-operatively on medications with the stimulation turned on. The median disease duration was 8 years for the DBS patients and 6 years for the control group, at which time point the precognitive assessments were performed.

The tests were assigned to 5 cognitive domains: attention and information processing speed (Stroop Colour-Word Test, Trail Making, and Digit Span), executive functions (Stroop ColourWord, Trail Making Test, and Wisconsin Card Sorting), fluency (semantic verbal fluency [VF] test, phonemic VF, and 5-point test), long-term memory (wordlist long-delayed recall, verbal learning: discrimination), working memory (Corsi blocks from the German version of the revised Wechsel Memory Scale, divided attention), and visuospatial functions (block design test, Rey Osterrieth Complex Figure Copy). Mood assessment was based on the UPDRS I, UPDRS II, and UPDRS IV for the analysis of neuropsychological measures, and $z$-scores (corrected for age, sex, and education) of each variable were averaged per each domain to obtain domain scores. Greater values represent better performance. The median follow-up range (at which time point the assessment occurred) was for both groups 24 months (Fig. 1).

\section{EEG Data}

For each patient in the DBS group, a 256-channel EEG of circa 15 min length was acquired at rest (Netstation 300; EGI Inc., Eugene, OR, USA, sampling rate $1,000 \mathrm{~Hz}$ ). Subjects were instructed to stay awake in a relaxed state. A semi-automated process was applied, in which each EEG recording was first visually inspected for sleep/sleepiness and EMG artefacts. The preselected segments were filtered, and artefacts due to eye movement, muscle contraction, drops in vigilance (sleep/sleepiness), electrodes, and ECG were removed further with MATLAB-based TAPEEG [27]. In the post-processing stage, 12 epochs of $4 \mathrm{~s}$ each were extracted from the cleaned EEG segments and spectral powers were calculated. To quantify EEG, spectral analysis is used to decompose a complex EEG signal into its component frequencies through Fourier transformation. Spectral power can be assessed globally (over the whole scalp) and over definite scalp regions. Using a high-density electrode system enables us to aggregate nearby signals thus potentially reducing noise. We obtained the spectral powers for the 10 regions of interest and also assessed spectral power globally. Thus, we analysed features in 6 frequency ranges and 11 locations, obtaining a total of 66 spectral features.

Relative power, which assesses the relative contribution of a particular frequency to the EEG signals, is calculated by dividing the absolute power in a given frequency band by the total power. We calculated median relative spectral powers in the following frequency ranges (Hz): 1-4 (delta), 4-8 (theta), 8-10 (alpha 1), 10-13 (alpha 2), 8-13 (alpha), and 13-30 (beta).

Electroencephalography Screening in DBS for Parkinson's Disease

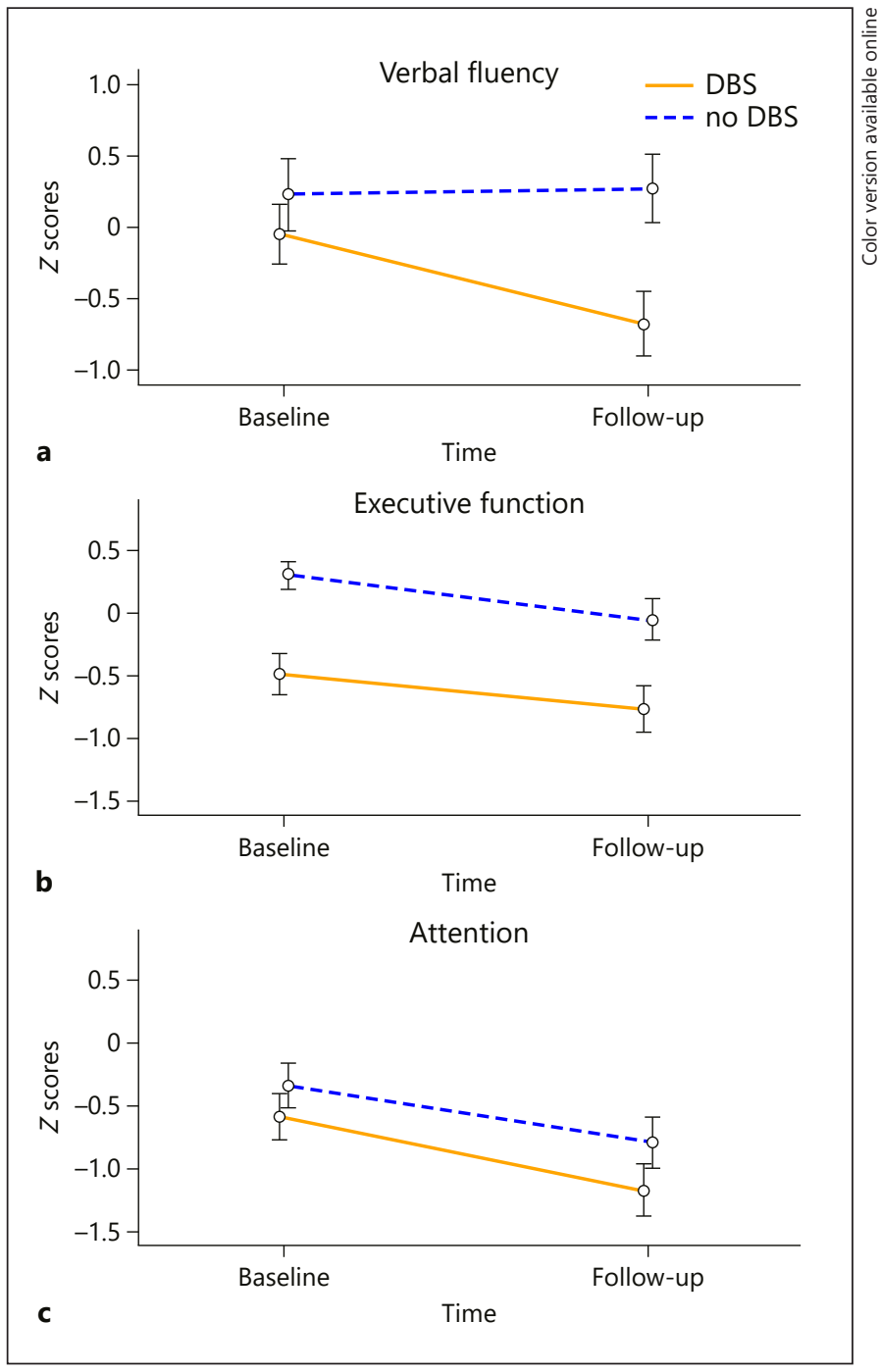

Fig. 1. $Z$-scores represent age-, sex-, and education-corrected and combined test scores (averaged per each domain to obtain domain scores). VF (a), executive function (b), and attention (c) of patients with PD treated with STN-DBS (DBS, orange line) versus treated conservatively (non-DBS, blue line) over 2 years. The performance in VF tests (a) in the DBS group was significantly reduced after 2 years $(p<0.05)$ as compared to the control group. No other interaction between time $\times$ group was statistically significant. DBS, deep brain stimulation; PD, Parkinson's disease; STN, subthalamic nucleus; VF, verbal fluency.

\section{Statistics}

Continuous variables were visually inspected for normality of distribution and statistically tested with the Kolmogorov-Smirnov test. In accordance with the results, parametric or nonparametric analyses were performed. All $p$ values $<0.05$ were considered statistically significant. MANOVA was used for a multivariate comparison for all selected difference scores between intervention conditions (DBS, non-DBS). 


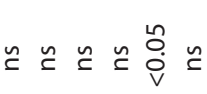

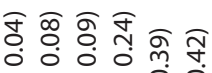

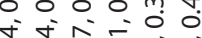

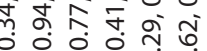

$\begin{array}{lllll}1 & 1 & 1 & 1 & 0\end{array}$

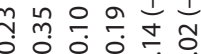

i i

궁

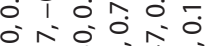

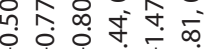

1 I 1 i I

둔

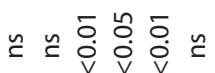

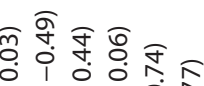

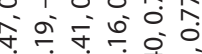

1 I

开

i

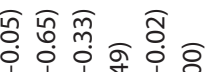

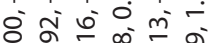

I I I

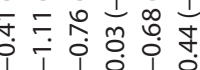

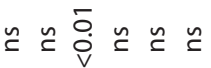

으 $\widehat{\bar{m}} \bar{m}$

i웅요

mิ

1. ב

过

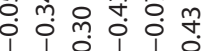

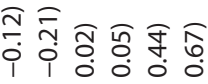

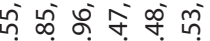

IIIIII

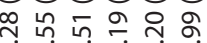

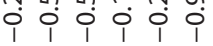

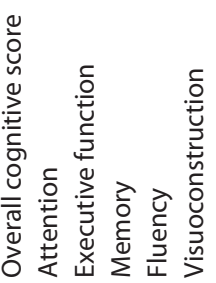

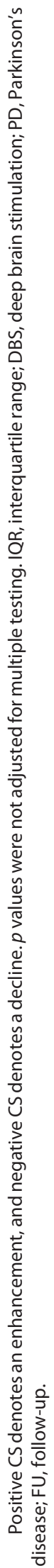

\section{Results}

The 2 groups did not differ in several sample characteristics or any variable regarding disease-related motor impairment at baseline (Table 1). At follow-up, scores on the UPDRS-II and UPDRS-III subscales did not significantly differ between the 2 PD groups.

In both groups (DBS and control groups), there was worsening of the overall cognitive score with a trend towards significance over time $(p$ time $=0.06)$ (Table 2). Attention declined significantly over time in both groups ( $p=0.005)$ without significant group differences. While the VF at baseline was the same in both groups, it significantly worsened on FU in the DBS group ( $p$ group $\times$ time $<0.05$ ). A decline over time was also seen in the executive function in both groups, with a stronger decline in the DBS group.

Looking at the baseline EEG and post-operative cognitive domains, we found strong (negative) correlations between the global relative power in the delta band at baseline with overall cognition $(r=-0.74, p<001)$, attention $(r=-0.52, p<0.05)$, and executive function $(r=-0.73$, $p<0.01$ ) domains post-DBS (Fig. 2a-c). Global power in the theta band at baseline was correlated with a post-operative decline in fluency $(r=-0.50, p<0.05)$. Global relative power in the alpha band had a strong positive correlation with executive function $(r=0.82, p<0.01)$ and overall cognition $(r=0.79, p<0.01)$ (Fig. 3a-c). In view of these findings, we wanted to further characterize the association between the EEG measures and cognition with the aid of linear models. By using a stepwise elimination procedure, the following variables were excluded from the model explaining post-operative overall cognition: age, gender, years of education, MMSE, motor symptoms (UPDRS III), disease duration, and LED. Delta power was significantly associated with the overall cog- 


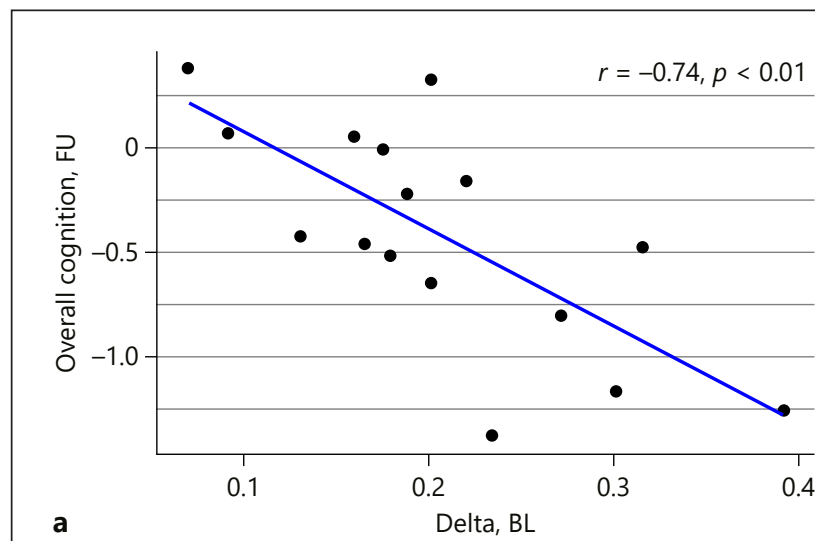

a

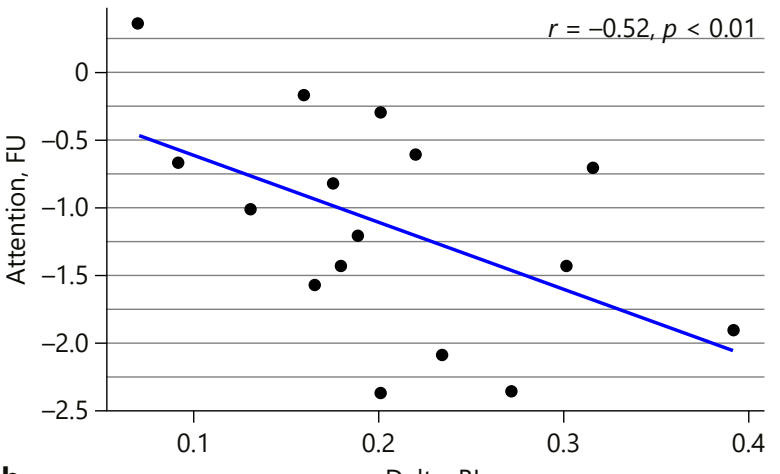

b

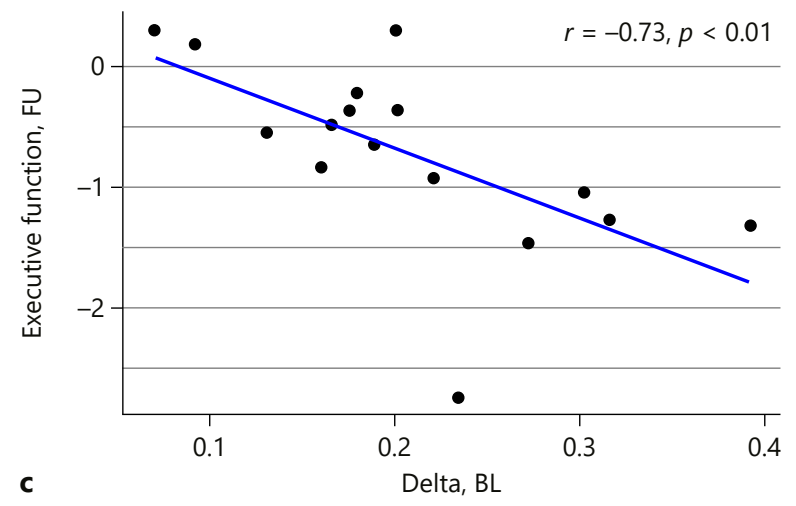

Fig. 2. a-c Spearman rank correlation coefficients $(r)$ between BL relative delta power $(1-4 \mathrm{~Hz})$ and $\mathrm{FU}$ cognition values in $N=16$ patients with DBS surgery in STN. $p$ values are adjusted by FDR correction. DBS, deep brain stimulation; STN, subthalamic nucleus; FDR, false discovery rate; BL, baseline; FU, follow-up.

nitive score $\left(p=0.00409\right.$, adjusted $\left.R^{2}=0.6341\right)$, which was not influenced by UPDRS III $(p=0.36817)$ and age $(p=0.06401)$. Global powers in the alpha 1 band $(8-10$ $\mathrm{Hz})$ were associated with executive function $(p=0.000134)$ with a high adjusted $R^{2}=$ of 0.8037 but confounded by MMSE scores $(p=0.000214)$. Alpha 1 power was associated with the attention domain $\left(p=0.0322, R^{2}=0.4538\right)$.

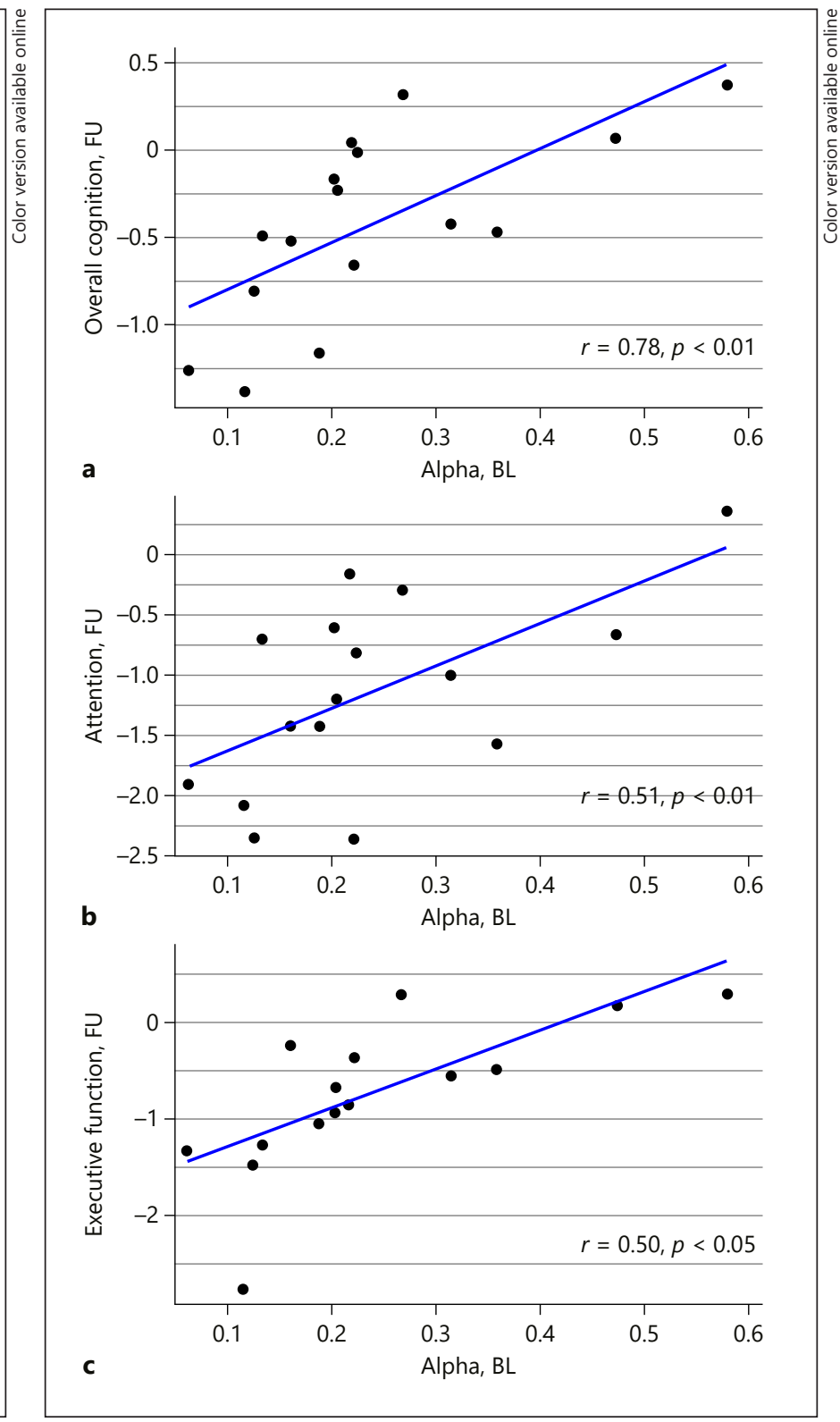

Fig. 3. a-c Spearman rank correlation coefficients $(r)$ between BL relative alpha power $(8-10 \mathrm{~Hz})$ and FU cognition values in $N=16$ patients with DBS surgery in STN. $p$ values are adjusted by FDR correction. DBS, deep brain stimulation; STN, subthalamic nucleus; FDR, false discovery rate; BL, baseline; FU, follow-up.

\section{Discussion}

In the present study, 2 groups of PD patients were compared regarding the prediction of EEG parameters on cognitive outcome after STN-DBS. The 2 groups were matched concerning age, gender, levodopa doses, disease duration, and motor impairment at baseline. 
Preoperative neuropsychological screening is still considered the gold standard for evaluating the cognitive status of PD patients and to assess eligibility for DBS surgery. Despite thorough preoperative neuropsychological screening, post-operative cognitive decline is still a major concern after DBS surgery [13-17]. One reason for this might be that neuropsychological testing may miss subtle neuropsychological deficits and insufficient cooperation of the patient in advanced PD may contribute to gaps in testing $[20,29]$. In contrast to neuropsychological testing, EEG requires minimal patient cooperation, can be rapidly performed, displays no learning effects, and is thus free of any potential test-retest bias [20]. More importantly, preclinical cortical and subcortical structural changes in PD not captured by routine neuropsychological testing may be detected by electrophysiological investigation and may account for the slowing of background EEG frequency [20].

Our study was based on extensive neuropsychological testing and high-resolution EEG data spanning global power in each of 6 frequency bands. In our study, the slowing of EEG in the delta power range significantly predicted overall worsening of cognition. We also found statistically significant correlations between delta power and attention as well as executive function post-DBS. Alpha 1 power was associated with the attention domain. Our results are in line with previous studies reporting a correlation of EEG slowing with cognitive impairment in PD [23, 24, 27, 30-32]. Preoperative (but standard) EEG was used for the assessment of the post-operative cognitive course after DBS, only once, to the best of our knowledge [24].

Markser et al. [24] reported cognitive deteriorations in 6 out of 30 patients with a lower median EEG frequency of $7.5 \mathrm{~Hz}$ (range 6.5-8.5), 4-12 months after STN-DBS. The remaining 24 patients who were post-operatively cognitively stable showed a higher preoperative median EEG frequency $(9 \mathrm{~Hz}$, range 7.5-11.5). The authors postulated that preoperative EEG may add useful information to the neuropsychological assessment to predict cognitive decline after electrode placement [24]. Multi-domain cognitive deterioration is a known complication of DBS, but outcomes are inconsistent across the reported studies, making it difficult to predict post-operative cognitive decline accurately [13-17]. The mechanisms as how DBS leads to cognitive deterioration are not fully understood but appear to be multifactorial [13-17]. Contributing factors for post-operative cognitive decline may relate to the natural progressive neurodegenerative course of $\mathrm{PD}$, chronic dopaminergic therapy, current spread from the dorsolateral motor STN section into the associative cognitive central portion of the nucleus [33], and post-operative medication reduction as well as surgery as itself $[13,16,34,35]$. Variability in electrode trajectories and targets within the STN, as well as in stimulation parameters, might partially explain the inconsistency [16, $17,33,36]$. Whether VF decline is a direct result of DBS is under debate $[32,37,38]$. Several mechanisms may account for VF decline such as medication dose, disease duration, and age. Parsons et al. [39] suggested that the important dopaminergic medication reduction that follows STN-DBS may account for depression and apathy, which in turn may lead to a decline in VF. Leimbach et al. [13] suggested a contrary hypothesis that VF decline may be the direct result of surgery and not an STN-DBS effect. Concerning attention, there was a significant decline over time in both groups, so this decline cannot be interpreted as a result of DBS surgery as such. The decline in VF was the only parameter which divided the DBS and non-DBS group over the follow-up period of 48 months and it is therefore exclusively attributable to the DBS surgery group. Moderate declines in VF are the most frequently observed neuropsychological declines in patients treated with STN-DBS [14, 17, 32].

In our study, the EEG could not predict specific cognitive changes secondary to DBS, specifically, VF decline. In our view, the purpose of preoperative EEG is not the prediction of specific cognitive changes induced by DBS but rather the preoperative assessment for potential vulnerability of PD patients for long-term cognitive decline. Standard neuropsychological testing can attest a normal cognitive state; however, the adjunctive preoperative EEG may show that the very same patient has slowing of background frequency, potentially a sign of long-term cognitive decline. The risk for cognitive impairment in PD within this context is 2 -fold, that is, through natural disease course and DBS. Accurate patient selection for DBS becomes more stringent. Cognitive dysfunction has also been reported as a complication of any type of surgery (in the brain or elsewhere), especially in the elderly; surgery and/or general anaesthesia might lead to the worsening of latent cognitive deficits that were not recognised preoperatively [35].

As DBS has the potential to lead to cognitive decline, tools for proper preoperative identification of patients particularly vulnerable for cognitive deterioration and in whom DBS might be inadvisable are of critical importance. The combination of a comprehensive neuropsychological assessment with EEG could improve the accuracy of preoperative screening [24].

A further important advantage of preoperative EEG is that it allows parallel assessment of increased but asymp- 
tomatic cerebral excitability. In a large cohort study, it was found that PD patients had a 1.7-fold increased risk of epileptic seizures compared to subjects without PD [40].

It must be pointed out however that preoperative EEG for the assessment of cognitive function requires particular attention to the state of wakefulness of the patient during the entire EEG recording. Sleep and sleepiness will cause background frequency slowing indistinguishable from that seen in cognitive impairment [41].

The strength of our study was that it was a matched, case-control study that included a comprehensive neuropsychological assessment and the semi-automated processing of high-resolution EEG, a non-invasive, universally available technique that can be applied in routine clinical practice. A further strength was that particular attention was paid to the highest quality EEG recordings by eliminating all segments affected by sleep/sleepiness. The limitations of our study were the relatively small sample size, the short follow-up period, and the absence of a wait list control group.

Our study was designed as a matched case-control study; however, we minimized the limitations of the study design by matching the control group with the DBS group on multiple parameters. The control group did not differ in age, gender, education, MMSE, LED, UPDRS, disease duration, and follow-up.

\section{Conclusion}

Screening for impaired cognitive function by using EEG could be of value for the early, preclinical detection of potential cognitive deficits in patients under consideration for DBS. Adding electroencephalographic data to the standard neuropsychological preoperative assessment may enable more accurate detection of particularly vulnerable patients for cognitive impairment with DBS and allow parallel assessment for increased, asymptomatic cerebral excitability. Further studies on the potential usefulness of EEG for predicting post-operative cognitive decline should employ larger prospective cohorts with longer follow-up.

\section{Acknowledgments}

We thank the psychology students Mrs Sarah Simmen, Mrs Sarah Trefzer, Mrs Sarina Roos, and Mrs Lisa Schiess in helping to collect the data. We thank Dr. Phillip Jaszczuk for proofreading the manuscript in the English language.

Electroencephalography Screening in DBS

for Parkinson's Disease

\section{Statement of Ethics}

The Joint Research Ethics Committee of the Cantons of Basel City and Basel Country (Ethik Kommission beider Basel) approved this study (EKBB ref. No. 135/11). All participants were fully informed of the nature of the study and gave their written informed consent.

\section{Conflict of Interest Statement}

The authors have no conflicts of interest to declare.

\section{Funding Sources}

There is no funding source for this study.

\section{Autor Contributions}

Christian Saleh: conception of the study, analysed the data, wrote the first draft, revised critically the final draft, and gave final approval for publication. Antonia Meyer analysed the data, wrote the draft, performed statistics, revised critically the final draft, and gave final approval for publication. Menorca Chaturvedi analysed the data, wrote the draft, revised critically the final draft, and gave final approval for publication. Selina Beltrani analysed the data, wrote the draft, revised critically the final draft, and gave final approval for publication. Ute Gschwandtner: conception of the study, analysed the data, wrote the draft, revised critically the final draft, and gave final approval for publication. Peter Fuhr: conception of the study, analysed the data, wrote the draft, revised critically the final draft, and gave final approval for publication.

\section{Data Availability Statement}

All data generated/analysed during this study are included in this article. Further enquiries can be directed to the corresponding author.

References

Dement Geriatr Cogn Disord 2021;50:349-356 DOI: 10.1159/000519053
1 Chaudhuri KR, Healy DG, Schapira AH; National Institute for Clinical Excellence. Nonmotor symptoms of Parkinson's disease: diagnosis and management. Lancet Neurol. 2006;5(3):235-45.

2 Jankovic J. Parkinson's disease: clinical features and diagnosis. J Neurol Neurosurg Psychiatry. 2008;79(4):368-76.

3 Poewe W, Gauthier S, Aarsland D, Leverenz JB, Barone P, Weintraub D, et al. Diagnosis and management of Parkinson's disease dementia. Int J Clin Pract. 2008;62(10):1581-7.

4 Sauerbier A, Jenner P, Todorova A, Chaudhuri KR. Non motor subtypes and Parkinson's disease. Parkinsonism Relat Disord. 2016;22(1):S41-6. 
5 Munhoz RP, Picillo M, Fox SH, Bruno V, Panisset M, Honey CR, et al. Eligibility criteria for deep brain stimulation in Parkinson's disease, tremor, and dystonia. Can J Neurol Sci. 2016;43(4):462-71.

6 Poewe W, Gauthier S, Aarsland D, Leverenz JB, Barone P, Weintraub D, et al. Diagnosis and management of Parkinson's disease dementia. Int J Clin Pract. 2008;62(10):1581-7.

7 Aarsland D, Creese B, Politis M, Chaudhuri KR, Ffytche DH, Weintraub D, et al. Cognitive decline in Parkinson disease. Nat Rev Neurol. 2017;13(4):217-31.

8 Svenningsson $\mathrm{P}$, Westman E, Ballard C, Aarsland D. Cognitive impairment in patients with Parkinson's disease: diagnosis, biomarkers, and treatment. Lancet Neurol. 2012; 11(8):697-707.

9 Martinez-Martin P, Forjaz MJ, Frades-Payo B, Rusinol AB, Fernandez-Garcia JM, BenitoLeon J, et al. Caregiver burden in Parkinson's disease. Mov Disord. 2007;22(7):924-31.

10 Petersen RC. Mild cognitive impairment: transition between aging and Alzheimer's disease. Neurologia. 2000;15(3):93-101.

11 Mao Z, Ling Z, Pan L, Xu X, Cui Z, Liang S, et al. Comparison of efficacy of deep brain stimulation of different targets in Parkinson's disease: a network meta-analysis. Front Aging Neurosci. 2019;11:23.

12 Honey CR, Hamani C, Kalia SK, Sankar T, Picillo M, Munhoz RP, et al. Deep brain stimulation target selection for Parkinson's disease. Can J Neurol Sci. 2017;44(1):3-8.

13 Leimbach F, Atkinson-Clement C, Wilkinson L, Cheung C, Jahanshahi M. Dissociable effects of subthalamic nucleus deep brain stimulation surgery and acute stimulation on verbal fluency in Parkinson's disease. Behav Brain Res. 2020;388:112621.

14 Limousin P, Foltynie T. Long-term outcomes of deep brain stimulation in Parkinson disease. Nat Rev Neurol. 2019;15(4):234-42.

15 Combs HL, Folley BS, Berry DT, Segerstrom SC, Han DY, Anderson-Mooney AJ, et al. Cognition and depression following deep brain stimulation of the subthalamic nucleus and globus pallidus pars internus in Parkinson's disease: a meta-analysis. Neuropsychol Rev. 2015;25(4):439-54.

16 Mehanna R, Bajwa JA, Fernandez H, Wagle Shukla AA. Cognitive impact of deep brain stimulation on Parkinson's disease patients. Parkinsons Dis. 2017;2017:3085140.

17 Witt K, Granert O, Daniels C, Volkmann J, Falk D, van Eimeren T, et al. Relation of lead trajectory and electrode position to neuropsychological outcomes of subthalamic neurostimulation in Parkinson's disease: results from a randomized trial. Brain. 2013;136(Pt 7):2109-19.
18 Babiloni C, De Pandis MF, Vecchio F, Buffo P, Sorpresi F, Frisoni GB, et al. Cortical sources of resting state electroencephalographic rhythms in Parkinson's disease related dementia and Alzheimer's disease. Clin Neurophysiol. 2011;122(12):2355-64.

19 Benz N, Hatz F, Bousleiman H, Ehrensperger MM, Gschwandtner U, Hardmeier M, et al. Slowing of EEG background activity in Parkinson's and Alzheimer's disease with early cognitive dysfunction. Front Aging Neurosci. 2014;6:314.

20 Klassen BT, Hentz JG, Shill HA, DriverDunckley E, Evidente VG, Sabbagh MN, et al. Quantitative EEG as a predictive biomarker for Parkinson disease dementia. Neurology. 2011;77(2):118-24.

21 Caviness JN, Hentz JG, Evidente VG, DriverDunckley E, Samanta J, Mahant P, et al. Both early and late cognitive dysfunction affects the electroencephalogram in Parkinson's disease. Parkinsonism Relat Disord. 2007;13(6): $348-54$.

22 Olde Dubbelink KT, Stoffers D, Deijen JB, Twisk JW, Stam CJ, Berendse HW. Cognitive decline in Parkinson's disease is associated with slowing of resting-state brain activity: a longitudinal study. Neurobiol Aging. 2013; 34(2):408-18.

23 Zimmermann R, Gschwandtner U, Hatz F, Schindler C, Bousleiman $\mathrm{H}$, Ahmed S, et al. Correlation of EEG slowing with cognitive domains in nondemented patients with Parkinson's disease. Dement Geriatr Cogn Disord. 2015;39(3-4):207-14.

24 Markser A, Maier F, Lewis CJ, Dembek TA Pedrosa D, Eggers C, et al. Deep brain stimulation and cognitive decline in Parkinson's disease: the predictive value of electroencephalography. J Neurol. 2015;262(10):2275-84.

25 Broggi G, Franzini A, Marras C, Romito L, Albanese A. Surgery of Parkinson's disease: inclusion criteria and follow-up. Neurol Sci. 2003;24(1):S38-40.

26 Coleman R, Ostrem J. Patient selection: when to consider deep brain stimulation for patients with Parkinson's disease, essential tremor, or dystonia. In: Marks W Jr, editor. Deep brain stimulation management. Cambridge: Cambridge University Press; 2010. p. 5-30.

27 Hardmeier M, Hatz F, Bousleiman H, Schindler C, Stam CJ, Fuhr P. Reproducibility of functional connectivity and graph measures based on the phase lag index (PLI) and weighted phase lag index (wPLI) derived from high resolution EEG. PLoS One. 2014;9(10):e108648.

28 R Core Team (2015). R: a language and Environment for Statistical Computing. Vienna: R Foundation for Statistical Computing; 2015. Available from: http://www.R-project.org/.
29 Geraedts VJ, Koch M, Contarino MF, Middelkoop HAM, Wang $\mathrm{H}$, van Hilten JJ, et al. Machine learning for automated EEG-based biomarkers of cognitive impairment during deep brain stimulation screening in patients with Parkinson's disease. Clin Neurophysiol. 2021;132(5):1041-8.

30 Cozac VV, Chaturvedi M, Hatz F, Meyer A, Fuhr P, Gschwandtner U. Increase of EEG spectral theta power indicates higher risk of the development of severe cognitive decline in Parkinson's disease after 3 years. Front Aging Neurosci. 2016;8:284.

31 Cozac VV, Gschwandtner U, Hatz F, Hardmeier M, Rüegg S, Fuhr P. Quantitative EEG and cognitive decline in Parkinson's disease. Parkinsons Dis. 2016;2016:9060649.

32 Cozac VV, Schwarz N, Bousleiman H, ChaturvediM,EhrenspergerMM, Gschwandtner $U$, et al. The verbal fluency decline after deep brain stimulation in Parkinson's disease: is there an influence of age? Mov Disord Clin Pract. 2016;3(1):48-52.

33 Alkemade A, de Hollander G, Miletic S, Keuken MC, Balesar R, de Boer O, et al. The functional microscopic neuroanatomy of the human subthalamic nucleus. Brain Struct Funct. 2019;224(9):3213-27.

34 Hanna-Pladdy B, Pahwa R, Lyons KE. Paradoxical effect of dopamine medication on cognition in Parkinson's disease: relationship to side of motor onset. J Int Neuropsychol Soc. $2015 ; 21(4): 259-70$.

35 Evered L, Scott DA, Silbert B, Maruff P. Postoperative cognitive dysfunction is independent of type of surgery and anesthetic. Anesth Analg. 2011;112(5):1179-85.

36 Guo S, Zhuang P, Hallett M, Zheng Z, Zhang Y, Li J, et al. Subthalamic deep brain stimulation for Parkinson's disease: correlation between locations of oscillatory activity and optimal site of stimulation. Parkinsonism Relat Disord. 2013;19(1):109-14.

37 Ehlen F, Schoenecker T, Kühn AA, Klostermann F. Differential effects of deep brain stimulation on verbal fluency. Brain Lang. 2014;134:23-33.

38 Hatz F, Meyer A, Roesch A, Taub E, Gschwandtner U, Fuhr P. Quantitative EEG and verbal fluency in DBS patients: comparison of stimulator-on and -off conditions. Front Neurol. 2018;9:1152. ;

39 Parsons TD, Rogers SA, Braaten AJ, Woods SP, Tröster AI. Cognitive sequelae of subthalamic nucleus deep brain stimulation in Parkinson's disease: a meta-analysis. Lancet Neurol. 2006;5(7):578-88.

40 Gruntz K, Bloechliger M, Becker C, Jick SS, Fuhr P, Meier CR, et al. Parkinson disease and the risk of epileptic seizures. Ann Neurol. 2018;83(2):363-74

41 Asadi-Pooya AA, Sperling MR. Normal awake, drowsy, and sleep EEG patterns that might be overinterpreted as abnormal. J Clin Neurophysiol. 2019;36(4):250-6. 